QUN CHEN, Ph.D.

Corresponding author

E-mail: chenqun631@csu.edu.cn

School of Traffic and Transportation Engineering,

Central South University

Changsha, 410075, China

YAN WANG, Ph.D. Candidate

E-mail: wangyanheroushi@163.com

School of Public Administration,

Central South University

Changsha, 410083, China
Traffic in the Cities

Original Scientific Paper

Submitted: July 4, 2014

Approved: Oct. 6, 2015

\title{
A MODEL FOR DYNAMIC PARKING CHOICE OF DRIVERS
}

\begin{abstract}
Taking time-varying parking demands and supplies into account, this paper develops a dynamic parking choice model to simulate drivers' searching for parking spaces between parking lots. In this study, each parking lot has one access passage and vehicles can move or wait for entering the park in the access passage. The flow propagation formula in the access passage of parking lots is established to obtain the number of vehicles entering the parking lot from the access passage within each time interval. This paper brings forward the instantaneous dynamic user-optimal condition of dynamic parking choice, constructs an equivalent variational inequality (VI) model and develops a diagonalization algorithm to solve the model. A numerical example demonstrates the rate of in-flow, the rate of out-flow, link flow, instantaneous travel time on each link or access passage of the parking lot, and the amount and routes of cruising traffic at each time point.
\end{abstract}

\section{KEYWORDS}

parking; dynamic; user-optimal; variational inequality (VI)

\section{INTRODUCTION}

In reality, drivers may need to search for a vacant parking space between parking lots in the vicinity of the destination. When the driver arrives at a park, they will park if vacant parking spaces are available at this parking lot; or else, they will assess the possible time necessary for waiting for a parking space to get vacated, and they will leave for another parking lot if the waiting time is too long. This paper models dynamic parking choice behaviours of drivers between parking lots, calculates the number of vehicles going into or out of each park at each time point, calculates link flows on the surrounding road networks, and finds out the routes of cruising traffic.
The dynamic traffic assignment focuses on route choice behaviours under time-varying traffic demands and time-varying traffic conditions. The models for dynamic traffic assignment can be classified into five categories according to research methods: Computer simulating [1, 2]; Mathematical programming [3, 4]; Optimal control theory [5, 6]; Variational inequality $(\mathrm{VI})$ and non-linear complementarity $[7,8$, 9, 10]; Fixed point theory [11]. The dynamic traffic assignment model based on computer simulating gives route choices of travellers by iteration, but it cannot guarantee the convergence and precision of solutions. The mathematical programming model also has many limitations because many dynamic traffic assignment problems cannot be converted into equivalent mathematical programming. The model based on optimal control theory has the advantage of good analysis, but in many cases the equivalent optimal control model to the user-optimal condition cannot be found. The models based on VI and non-linear complementarity have been developed in recent years; they have a simple form and can be easily solved. In order to more accurately simulate the dynamics characteristics of the network traffic flow, Lo and Szeto in [12] introduced the Cell Transmission Model (CTM) and constructed a dynamic traffic assignment model based on CTM using VI. Ramadurai and Ukkusuri in [13] proposed a dynamic user equilibrium model for combined activity-travel choices using activity-travel supernetwork representation, and a cell-based transmission model (CTM) is embedded to capture the traffic flow dynamics. Tian et al. in [14] presented a cumulative perceived value-based dynamic user equilibrium model by applying the prospect theory to formulate the travellers' risk evaluation on arrival time, and considered departure time and route choices simultaneously.

Although the dynamic assignment problems have been studied by a lot of researchers, these studies 
did not take parking choice at the end of the trip into account in modelling the dynamic route choice behaviours of the drivers. This has encouraged the carrying out of this study. The dynamic parking choice model simulates drivers' searching for the parking spaces between parking lots, and considers not only road networks but also parking at the parking lots, so it extends the dynamic traffic assignment theory which has been studied by a lot of researchers $[8,9,15]$.

\section{NOTATIONS AND DEFINITIONS}

The road network is denoted by $G=(N, A)$, where $N$ is the set of all nodes, $A$ is the set of all links; $s$ denotes destination node; $a=(I, m)$ is the link with starting node $I$ and end node $m$. $E_{l}$ is the set of links pointing at node I; $F$ is the set of links out of node $I$, see Figure 1.

Figure 2 shows the park and its access passage, and this paper supposes that each park has and only has one access passage, and that vehicles can move or wait for entering the park in its access passage.

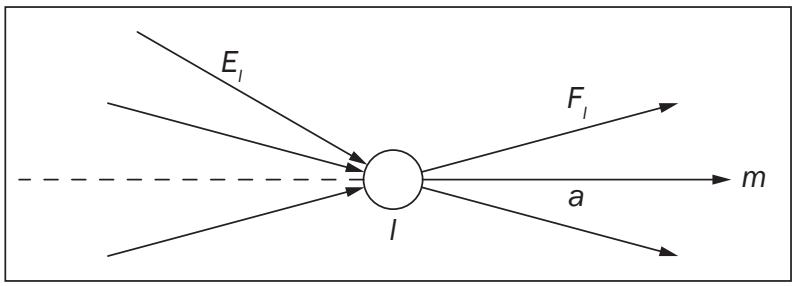

Figure 1 - Relation between links and nodes

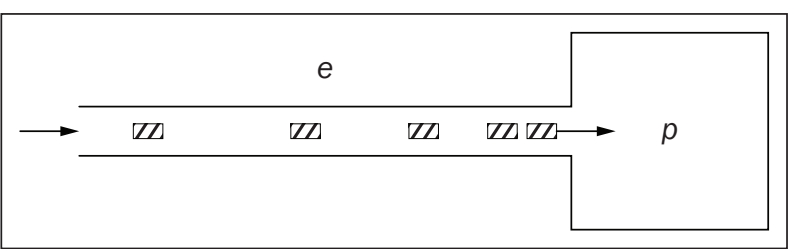

Figure 2 - The park and its access passage

The time range is $[0, T]$, which should be long enough to make a traveller be able to complete their trip in this time interval. For $t \in[0, T]$, the following variables will be introduced.

$u_{a}(t)$ - Rate of in-flow of link a (i.e. the number of vehicles entering link a) at time point $t$, and $u_{a}^{s}(t)$ is the rate of in-flow of link $a$ at time point $t$ whose destination is $s$, $u_{a}(t)=\sum_{s} u_{a}^{s}(t)$

$v_{a}(t)$ - Rate of out-flow of link a (i.e. the number of vehicles coming out of link a) at time point $t$, and $v_{a}^{s}(t)$ is the rate of out-flow of link $a$ at time point $t$ whose destination is $s$, $v_{a}(t)=\sum_{s} v_{a}^{s}(t)$.

$x_{a}(t)$ - Flow on link a (number of vehicles) at time point $t$, and $x_{a}^{s}(t)$ is the flow (number of vehicles) on link $a$ at time point $t$ whose destination is $s, x_{a}(t)=\sum_{s} x_{a}^{s}(t)$.

$g_{1}^{s}(t)-$ Flow produced at node $I$ at time point $t$ whose destination is $\mathrm{s}$.

$c_{a}(t)$ - Instantaneous travel time or reactive travel time on link $a$ at time point $t$, also has a form of $c_{a}\left[x_{a}(t), u_{a}(t), v_{a}(t)\right]$.

$\eta_{1}^{s}(t)$ - Minimal instantaneous path travel time from node $/$ to destination $s$.

$\tau_{a}(t)$ - Actual travel time on link a of the vehicle entering the link at time point $t$, which is also influenced by the followed vehicles.

$\chi_{p}^{s}(t)$ - Parking duration of the vehicle entering park $p$ at time point $t$ whose destination is $s$.

$Q_{p}$ - The number of parking spaces in park $p$.

$B_{p, s}$ - The time for walking egress trip from park $p$ to destination s.

$C_{e}$ - The maximal number of vehicles existing in passage $e$ at the same time.

$D_{e}$ - The free travel time of vehicles in passage e.

$\hat{x}_{p}(t)$ - The number of vehicles parking at park $p$ at time point $t . \hat{x}_{p}^{s}(t)$ is the number of vehicles parking at park $p$ at time point $t$ whose destination is $s, \hat{x}_{p}(t)=\sum_{s} \hat{x}_{p}^{s}(t)$.

$\hat{u}_{p}(t)$ - Rate of in-flow of park $p$ (i.e. the number of vehicles entering park $p$ ) at time point $t$, and $\hat{u}_{p}^{s}(t)$ is the rate of in-flow of park $p$ at time point $t$ whose destination is $s$, $\hat{u}_{p}(t)=\sum_{s} \hat{u}_{p}^{s}(t)$.

$\hat{v}_{p}(t)$ - Rate of out-flow of park $p$ (i.e. the number of vehicles leaving park $p$ ) at time point $t$, and $\hat{v}_{p}^{s}(t)$ is the rate of out-flow of park $p$ at time point $t$ whose destination is $s$, $\hat{v}_{p}(t)=\sum_{s} \hat{v}_{p}^{s}(t)$.

\section{FLOW PROPAGATION FORMULA AT PARKING LOTS}

The time range $[0, T]$ is discretized into $K+1$ equally small time intervals $0,1,2, \ldots, K$. The starting point of time interval $k(0 \leq k \leq K)$ is also named as time point $k$, while the end point of time interval $k$ is just the starting point of time interval $k+1$. The free-flow travel time on each link or passage should be larger than or equal to one small time interval and parking duration of each vehicle in the park should also be larger than or equal to one small time interval.

Figure 3 shows a simple road and park network. Node 1 is the origin; nodes 4 and 5 denote two parks; $2 \rightarrow 4$ is the access passage of park $4 ; 3 \rightarrow 5$ is the access passage of park $5 ; 1 \rightarrow 2,1 \rightarrow 3,2 \rightarrow 3,3 \rightarrow 2$ are road links; especially $2 \rightarrow 3,3 \rightarrow 2$ link park 4 with 5 , and drivers can choose between parks 4 and 5 via links $2 \rightarrow 3,3 \rightarrow 2$. 


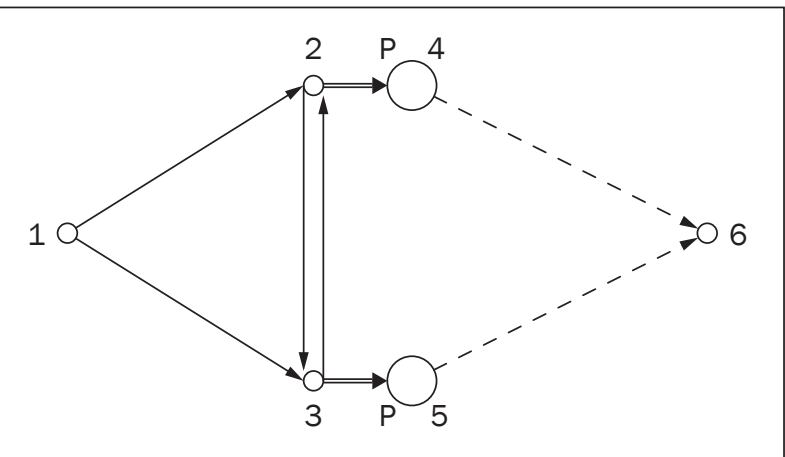

Figure 3 - Park and road network

Phase update formula of the number of vehicles parking at park $p$ is

$\hat{x}_{p}^{s}(k)=\hat{x}_{p}^{s}(k-1)+\hat{u}_{p}^{s}(k-1)-\hat{v}_{p}^{s}(k-1)$,

$\forall p, s ; k=1,2, \cdots, k$

For park $p$, destination $s$ and time interval $k, \hat{i}_{p, k}^{s}$ is a time point that satisfies $\hat{i}_{p, k}^{s}+\chi_{p}^{s}\left(\hat{i}_{p, k}^{s}\right)=k$. All the values of $\hat{i}_{p, k}^{s}$ construct the set $\hat{i}_{p, k}^{s}$. The flow propagation constraint of park $p$ is

$$
\sum_{i_{p, p}^{s} \in \epsilon_{p, k}^{s}} \hat{u}_{p}^{s}\left(\hat{i}_{p, k}^{s}\right)=\hat{v}_{p}^{s}(k), \forall p, s, k
$$

Phase update formula of the number of vehicles in passage $e$ is

$x_{\mathrm{e}}^{\mathrm{s}}(k)=x_{\mathrm{e}}^{\mathrm{s}}(k-1)+u_{\mathrm{e}}^{\mathrm{s}}(k-1)-v_{\mathrm{e}}^{\mathrm{s}}(k-1)$,

$\forall e, s ; k=1,2, \cdots, k$

$v_{e}^{s}(k)$ is analysed based on the following suppositions (1)-(5).

1) Each park has and only has one access passage.

2) Overtaking is not permitted in the access passage of the park, i.e. it satisfies the first-in-firstout (FIFO) rule which denotes that the vehicle entering the passage first will be the first to enter the park.

3) Stop-and-go driving may occur because of vehicles queuing in the access passage of the park. Here, the starting acceleration time and braking deceleration time of vehicles in the passage will be ignored. And the speed of vehicles moving in the passage is regarded as fixed.

4) Vehicles in the passage only queue waiting to enter the parking lot not including the queuing from disturbance of traffic flows.

5) All queuing vehicles stop or move forward one by one at a known minimum safe distance when line-up appears in the passage.

The following analysis supposes that the park has enough vacant parking spaces. Figure 4 shows the park and its access passage.

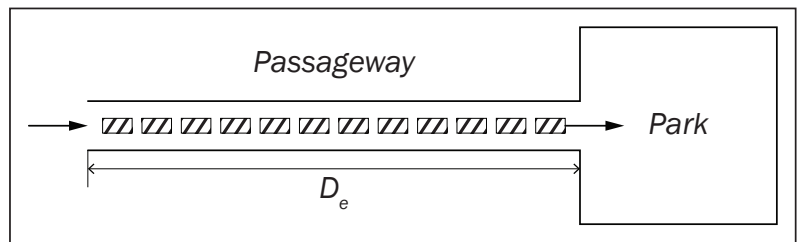

Figure 4 - The park and its access passage

Suppose that the free travel time (without queuing to wait) of vehicles in the passage is $D_{e}$ time intervals. Since the passage length is known, the maximal number of queuing vehicles that the passage can accommodate at the same time is $C_{e}$ according to supposition (5) that a fixed safe distance lies between immediate vehicles when they are queuing in the passage. The distance covered by the moving vehicle within one time interval is $d_{\mathrm{e}}$, so the maximal number of vehicles existing in the space interval with length $d_{e}$ at the same time is $\frac{C_{e}}{D_{e}}$ if they are queuing to wait.

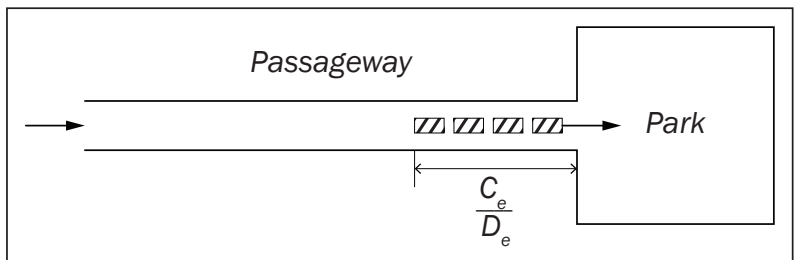

Figure 5 - The number of queuing vehicles in the space interval with length $d_{e}$

$x_{e}(k)=u_{e}(k-1)+u_{e}(k-2)+\ldots+u_{e}\left[k-\left(D_{e}-1\right)\right]+\ldots+$ $+u_{e}(0)-v_{e}(k-1)-v_{e}(k-2)-\ldots-v_{e}(0)$

At the start of time interval $k$, the farthest distance that vehicles $u_{e}(k-1)$ go in the passage is $d_{e}$, the farthest distance that vehicles $u_{e}(k-2)$ go in the passage is $2 d_{e}$, $\ldots$, the farthest distance that vehicles $u_{e}\left[k-\left(D_{e}-1\right)\right]$ go in the passage is $\left(D_{\mathrm{e}}-1\right) d_{\mathrm{e}}$. At the end of time interval $k$, the farthest distance that vehicles $u_{e}\left[k-\left(D_{e}-1\right)\right]$ go in the passage is $D_{e} d_{e}$ (i.e. passage length), and the vehicle entering first the passage among vehicles $u_{e}\left[k-\left(D_{e}-1\right)\right]$ travels a space interval with length $D_{e} d_{e}$ in the passage (i.e. the whole passage length), and is about to leave but has not yet left the passage. So, according to first-in-first-out (FIFO) rule, vehicles $u_{e}(k-1), u_{e}(k-2), \ldots, u_{e}\left[k-\left(D_{e}-1\right)\right]$ have been staying in the passage by the end of time interval k. So, in time interval $k$, the vehicles which may leave the passage and then enter the park should be part of $u_{e}\left(k-D_{e}\right)$, $u_{e}\left[k-\left(D_{e}+1\right), \ldots, u_{e}(0)\right.$, the remaining vehicles in the passage among which at the start of time interval $k$ (most of which have entered the park before interval $k$ ) are $x_{e}(k)-\left\{u_{e}(k-1)+u_{e}(k-2)+\ldots+u_{e}\left[k-\left(D_{e}-1\right)\right]\right\}$.

Proposition 1:

After a vehicle starts queuing to wait (from moving to queuing) in the passage, it always stops or goes forward close to the nearest vehicle in the front with minimum safe interval until it enters the park. 
Proof:

According to supposition (3) that the acceleration time and braking time of vehicles in the passage are ignored, after a vehicle starts queuing to wait from moving in the passage (the queuing vehicle keeps minimum safe distance from the nearest vehicle in the front), if the vehicle in the front moves, then the following queuing vehicle also moves at the same speed; if the vehicle in the front stops, then the following vehicle also stops to keep the minimum safe interval. Thus, it always stops or goes forward close to the nearest vehicle in the front with the minimum safe distance until it enters the park, and no bigger interval than the minimum safe distance between them occurs.

Proposition 2:

According to supposition (4) that vehicles in the passage only queue waiting to enter the parking lot without the presence of queuing from disturbance of traffic flows, if queuing occurs in the access passage of the park, then the queue starts from the gate (i.e. the link site of the park and its access passage) of the park and extends backwards along the passage.

Proof:

If a vehicle in the passage queues to wait while the vehicle in front of it moves instead of queuing to wait, then according to supposition (3) that acceleration time and braking time of vehicles in the passage are ignored, if a vehicle moves then the following vehicle also moves with the same speed instead of stopping. Here a contradiction occurs, so the vehicle in front of a queuing vehicle must be in the situation of queuing to wait, i.e. the queue starts from the gate (i.e. the link site of the park and its access passage) of the park and extends backwards along the passage.

Theorem 1

At time point $k$, if

$x_{e}(k)-\left\{u_{e}(k-1)+u_{e}(k-2)+\cdots+u_{e}\left[k-\left(D_{e}-1\right)\right]\right\}>\frac{C_{e}}{D_{e}}$ then the last vehicle entering the park among vehicles $u_{e}\left(k-D_{e}\right)$ must have queued (i.e. stopped) at one time point before. The number of vehicles leaving the passage and entering the park in one time interval is $\frac{C_{e}}{D_{e}}$ under ignoring acceleration time of vehicles and assuming the park has enough vacant parking spaces.

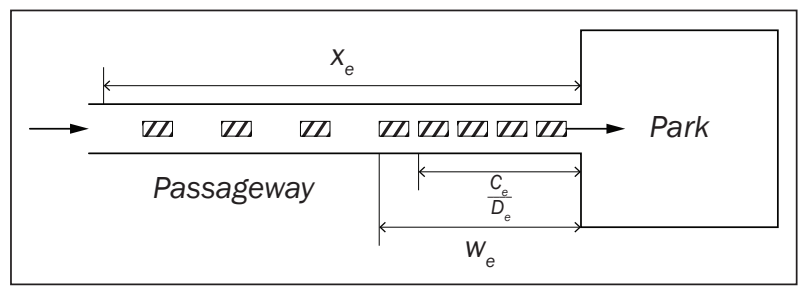

Figure 6 - The case when the number of queuing vehicles is larger than $\frac{C_{e}}{D_{e}}\left(w_{e}\right.$ is the number of queuing vehicles,

$$
\left.w_{e}=x_{e}(k)-\left\{u_{e}(k-1)+u_{e}(k-2)+\ldots+u_{e}\left[k-\left(D_{e}-1\right)\right]\right\}\right)
$$

Proof:

Let us assume the opposite that, at time point $k$, the last vehicle entering the passage among $u_{e}\left(k-D_{e}\right)$ has never queued to wait for parking before, then the distance it travels is greater than or equal to $\left(D_{e}-1\right) d_{e}$ and less than or equal to $D_{e} d_{e}$ at the start of time interval $k$ or at the end of time interval $k-1$. So, according to the FIFO condition, all the remaining vehicles in the passage among $u_{e}\left(k-D_{e}\right), u_{e}\left[k-\left(D_{e}+1\right)\right], \ldots, u_{e}(0)$ lie in the space interval with length $d_{e}$ from the gate of the park at time point $k$. However, the maximal number of vehicles the space interval with length $d_{e}$ from the gate of the park in the passage can accommodate is $\frac{C_{e}}{D_{e}}$,
while $x_{e}(k)-\left\{u_{e}(k-1)+u_{e}(k-2)+\cdots+u_{e}\left[k-\left(D_{e}-1\right)\right]\right\}>\frac{C_{e}}{D_{e}}$ so there is a contradiction. Thus, the last vehicle into the park among $u_{\mathrm{e}}\left(k-D_{\mathrm{e}}\right)$, which is also the last vehicle of $x_{e}(k)-\left\{u_{e}(k-1)+u_{e}(k-2)+\ldots+u_{e}\left[k-\left(D_{e}-1\right)\right]\right\}$, must have queued to wait at one time point before. According to proposition 2, the queue starts from the gate of the park and extends backwards along the passage, i.e. all vehicles in front of it are also queuing; and according to proposition 1, a vehicle which has ever queued to wait for parking must afterwards stop or go forward close to the nearest vehicle in the front with minimum safe distance until it enters the park. So after this time point, all vehicles of $x_{e}(k)-\left\{u_{e}(k-1)+u_{e}(k-2)+\ldots+u_{e}\left[k-\left(D_{e}-1\right)\right]\right\}$ queue or move forward keeping a minimum safe distance from the vehicle in the front. It is obvious from Figure 6 that the number of vehicles leaving the passage and entering the park in one time interval is just the num$\operatorname{ber}\left(\frac{C_{e}}{D_{e}}\right)$ of queuing vehicles in the space interval with length $d_{\mathrm{e}}$ which the vehicle travels in one time interval. Theorem 2

At time point $k$, if

$x_{e}(k)-\left\{u_{e}(k-1)+u_{e}(k-2)+\cdots+u_{e}\left[k-\left(D_{e}-1\right)\right]\right\} \leq \frac{C_{e}}{D_{e}}$

then vehicles $u_{e}\left(k-D_{e}\right)$ all lie in the space interval with length $d_{e}$ from the gate of the park. Vehicles $x_{e}(k)-\left\{u_{e}(k-1)+u_{e}(k-2)+\ldots+u_{e}\left[k-\left(D_{e}-1\right)\right]\right\}$ can all 
go into the park in the time interval $k$ under ignoring the acceleration time of the vehicle and assuming the park has enough vacant parking spaces.

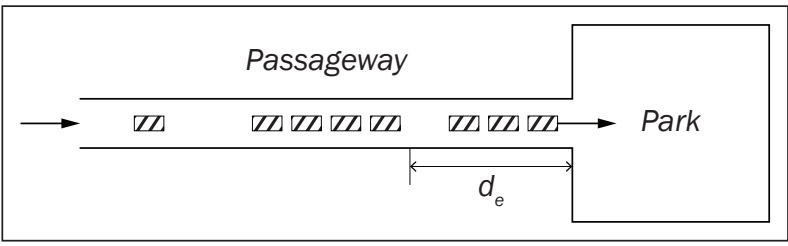

Figure 7 - The case when the number of queuing vehicles is less than or equal to $\frac{C_{e}}{D_{e}}$

Proof:

At time point $k$, if a vehicle of $u_{e}\left(k-D_{e}\right)$ (suppose it is the last vehicle going into the passage among $\left.u_{e}\left(k-D_{e}\right)\right)$ is outside the space interval with length $d_{e}$ from the gate of the park, then it must have queued to wait at one time point before because if it has never queued for parking, it would have already been in the space interval with length $d_{\mathrm{e}}$ from the gate of the park at time point $k$. According to propositions 1 and 2 , the queuing vehicle must afterwards stop or go forward close to the nearest vehicle in the front with minimum safe distance until it enters the park, and vehicles queuing or moving at intervals of minimum safe distance keeps from it to the gate of the park. However, $x_{e}(k)-\left\{u_{e}(k-1)+u_{e}(k-2)+\cdots+u_{e}\left[k-\left(D_{e}-1\right)\right]\right\} \leq \frac{C_{e}}{D_{e}}$ which means that queuing vehicles are all in the space interval with length $d_{e}$ from the gate of the park because the space interval with length $d_{e}$ in the passage can accommodate $\frac{C_{e}}{D_{e}}$ queuing vehicles, which contradicts the supposition that the vehicle which has ever queued to wait for parking at one time point before or the last vehicle into the passage among $u_{e}\left(k-D_{e}\right)$ is outside the space interval with length $d_{e}$ from the gate of the park. Thus, vehicles $u_{e}\left(k-D_{e}\right)$ should all lie in the space interval with length $d_{e}$ from the gate of the park. According to the FIFO condition, the remaining vehicles in the passage among $u_{e}\left[k-\left(D_{e}+1\right)\right], \ldots, u_{e}(0)$ should also be in the space interval with length $d_{e}$ from the gate of the park. Since all vehicles in the space interval with length $d_{e}$ in the passage can go into the park within one time interval, so vehicles $x_{e}(k)-\left\{u_{e}(k-1)+u_{e}(k-2)+\ldots+u_{e}\left[k-\left(D_{e}-1\right)\right]\right\}$ can all enter the park in time interval $k$ under ignoring acceleration time of vehicles and assuming the park has enough vacant parking spaces (See Figure 7).

So, synthetically from theorems 1 and 2 , the number of vehicles entering the park from the passage in time interval $k$ is

$$
\begin{aligned}
& v_{e}(k)=\min \left(x_{e}(k)-\left\{u_{e}(k-1)+u_{e}(k-2)+\right.\right. \\
& \left.\left.+\cdots+u_{e}\left[k-\left(D_{e}-1\right)\right]\right\}, \frac{C_{e}}{D_{e}}\right)
\end{aligned}
$$

The above analysis is on the basis of the supposition that the park has enough vacant parking spaces. However, if the number of vacant parking spaces in park $p$ connected with passage $e$ is given, the restriction of the number of vacant parking spaces needs to be taken into account. The number of vacant parking spaces in time interval $k$ is $Q_{p}-\hat{x}_{p}(k)+\hat{v}_{p}(k)$, so,

$$
\begin{aligned}
& v_{e}(k)=\min \left(\operatorname { m i n } \left(x_{e}(k)-\left\{u_{e}(k-1)+u_{e}(k-2)+\right.\right.\right. \\
& \left.\left.\left.+\cdots+u_{e}\left[k-\left(D_{e}-1\right)\right]\right\}, \frac{C_{e}}{D_{e}}\right), Q_{p}-\hat{x}_{p}(k)+\hat{v}_{p}(k)\right)
\end{aligned}
$$

$v_{e}^{s}(k)$ may be determined after $v_{e}(k)$ is known.

As seen in Figure 8, according to the supposition that the free flow travel time on the passage is larger than or equal to one time interval, so any of $u_{e}(k)$ would not leave the passage in the time interval $k$. At time point $k$, the vehicles which have entered the passage are $u_{e}(k-1), u_{e}(k-2), \ldots, u_{e}(1), u_{e}(0)$. At the start of time interval $k$,

$\sum_{\lambda=0}^{k-1} v_{e}(\lambda)=\sum_{\lambda=0}^{k-1} v_{p}(\lambda)+x_{p}(k)=u_{e}(0)+$

$+u_{\mathrm{e}}(1)+\cdots+u_{\mathrm{e}}\left(k_{2}-1\right)+\alpha u_{\mathrm{e}}\left(k_{2}\right)$

at the end of time interval $k$,

$\sum_{\lambda=0}^{k} v_{e}(\lambda)=u_{e}(0)+u_{e}(1)+\cdots+u_{e}\left(k_{1}-1\right)+\beta u_{e}\left(k_{1}\right)$

where $k_{1}$ and $k_{2}$ are the sequence numbers of time intervals between 0 and $k-1$ and $k_{1} \geq k_{2}, 0 \leq \alpha \leq 1$, $0 \leq \beta \leq 1$.

$$
\begin{aligned}
& v_{\mathrm{e}}(k)=\sum_{\lambda=0}^{k} v_{\mathrm{e}}(\lambda)-\sum_{\lambda=0}^{k-1} v_{\mathrm{e}}(\lambda)=\beta u_{\mathrm{e}}\left(k_{1}\right)+ \\
& +u_{\mathrm{e}}\left(k_{1}+1\right)+\cdots+(1-\alpha) u_{\mathrm{e}}\left(k_{2}\right)
\end{aligned}
$$

\begin{tabular}{|c|c|c|c|c|c|c|c|c|}
\hline & & & \multicolumn{6}{|c|}{$\sum_{\lambda=0}^{k} v_{e}(\lambda)$} \\
\hline$u_{e}(k-1)$ & $u_{e}(k-2)$ & ... & $u_{e}\left(k_{1}\right)$ & $\ldots$ & $u_{e}\left(k_{2}\right)$ & $\ldots$ & $u_{e}(1)$ & $u_{e}(0)$ \\
\hline & & & & \multicolumn{2}{|c|}{$v_{e}(k)$} & \multicolumn{3}{|c|}{$\sum_{\lambda=0}^{k-1} v_{e}(\lambda)$ (i.e. $\left.\sum_{\lambda=0}^{k-1} v_{p}(\lambda)+x_{p}(k)\right)$} \\
\hline
\end{tabular}

Since vehicles $u_{\mathrm{e}}^{\mathrm{s}}(k-1), \ldots, u_{\mathrm{e}}^{\mathrm{s}}(1), u_{\mathrm{e}}^{\mathrm{s}}(0)$ of $u_{\mathrm{e}}(k-1)$, $u_{e}(k-2), \ldots, u_{e}(1), u_{e}(0)$ are all known, $v_{e}^{s}(k)$ of $v_{e}(k)$ can also be known.

Figure 8 - Flow analysis in the passage 
In the joint of park $p$ and its access passage $e$,

$\hat{u}_{p}^{s}(k)=v_{e}^{s}(k)$

$u_{e}(k)$ is analysed as follows.

There is also a limit on the maximal number of vehicles which can enter the access passage of the park. If the passage has enough spaces, the maximal number of vehicles which can go into the passage within one time interval is $\frac{C_{e}}{D_{e}}$, since vehicles are supposed to move at a fixed speed in the passage and the maximal distance the vehicle can go within one time interval is $d_{e}$ and the maximal number of queuing vehicles existing in the space interval with length $d_{\mathrm{e}}$ at the same time is $\frac{C_{e}}{D_{e}}$. If the restriction of spaces available in the passage is taken into account, the number of vehicles which can go into the passage should be less than or equal to the maximal number of vehicles $\left(C_{e}-x_{e}(k)+v_{e}(k)\right)$ that the vacant spaces in the pas sage can accommodate. So, the number of vehicles which can go into the passage in time interval $k$ must be less than or equal to $\min \left(\frac{C_{e}}{D_{e}}, C_{e}-x_{e}(k)+v_{e}(k)\right)$.

\section{VI MODEL FOR DYNAMIC PARKING CHOICE}

\subsection{Dynamic user-optimal (DUO) condition of dynamic parking choice based on instantaneous travel time}

Instantaneous dynamic user-optimal condition of dynamic parking choice: during the time range $[0, T]$, at any time point and at any decision node (road network nodes or the joint of links and access passage of the park), if the instantaneous travel time on all the used paths are equal and equal to the minimum instantaneous travel time, while the instantaneous travel time on all unused paths is not less than the minimum instantaneous travel time, then the flows on the networks are subject to the DUO condition based on instantaneous travel time.

$u_{a}^{s}(t)>0$ means that link $a=(I, m)$ is used by the traveller whose destination is s at time point $t ; u_{\mathrm{e}}^{s}(t)>0$ means that the passage $e$ and the corresponding park are chosen by the traveller whose destination is $s$ at time point $t$. $\pi^{\text {ls }}$ is a path from decision node / to destination node $s, \phi_{1 \pi^{s}}^{s}(t)$ is the instantaneous travel time on path $\pi^{\text {ls }}$ at time point $t$.

$\phi_{1, \pi^{s}}^{\mathrm{s}}(t)=\sum_{a \in \pi^{s}} c_{a}(t)+B_{p, s}, \quad(p, s) \in \pi^{l s}$

where (7) means that the travel time on a path from decision node $/$ to destination node $s$ includes travel time on all links and access passage of the park of this path, and the walking time from the park to the destination, $\eta_{l}^{\mathrm{s}}(t)=\min _{\pi^{s}}\left\{\phi_{l, \pi^{s}}^{\mathrm{s}}(t)\right\}$.

The DUO condition based on instantaneous travel time can be written as (8) (13). For road link a,

$\left[\eta_{m}^{s}(t)+c_{a}(t)-\eta_{1}^{s}(t)\right] u_{a}^{s}(t)=0, \forall a, s, t$

$\eta_{m}^{\mathrm{s}}(t)+c_{a}(t) \geq \eta_{1}^{\mathrm{s}}(t), \quad \forall a, s, t$

$u_{a}^{s}(t) \geq 0, \quad \forall a, s, t$

It is known from (8), (9), (10) that if $u_{a}^{s}(t)>0$, then $\eta_{m}^{s}(t)+c_{a}(t)=\eta_{1}^{s}(t)$, which means that if road link a is chosen by the traveller whose destination is $s$, then road link a must be on the shortest path between nodes $I$ and $s$; and if $u_{a}^{s}(t)=0$, then $\eta_{m}^{s}(t)+c_{a}(t) \geq \eta_{1}^{s}(t)$.

For the passage $e=(I, m)(I$ is the start node of the passage, for example, nodes 2 and 3 in Figure $3 ; m$ is the park), the DUO condition with capacity constraints can be defined as (see literature [16])

$\eta_{m}^{s}(t)+c_{e}(t)+\delta_{e}(t)\left\{\begin{array}{ll}=\eta_{1}^{s}(t), & \text { if } u_{e}^{s}(t)>0 \\ \geq \eta_{1}^{s}(t), & \text { if } u_{e}^{s}(t)=0\end{array} \forall e, s, t\right.$

where $\delta_{e}(t)$ can be regarded as "virtual travel time" caused by the capacity constraint of passage $e$.

$\delta_{e}(t)\left\{\begin{array}{l}\geq 0, \text { if } \sum_{s} u_{e}^{s}(t)=u_{e m}(t) \\ =0, \text { if } \sum_{s} u_{e}^{s}(t)<u_{e m}(t)\end{array} \forall e, t\right.$

where

$u_{\mathrm{em}}(t)=\min \left(\frac{C_{e}}{D_{e}}, C_{e}-x_{e}(t)+v_{e}(t)\right)$

$\eta_{m}^{s}(t)$ is the walking time from the chosen park to the destination, and a value independent of $t$. If $\sum_{s} u_{e}^{s}(t)<u_{e m}(t)$ then $\delta_{e}(t)=0$ and the capacity constraint does not work; if $\sum_{\mathrm{s}} u_{\mathrm{e}}^{\mathrm{s}}(t)=u_{\mathrm{em}}(t)$ and $\eta_{m}^{s}(t)+c_{e}(t)=\eta_{l}^{s}(t)$, then $\delta_{e}(t)=\stackrel{s}{0}$;

and if $\sum_{s} u_{e}^{s}(t)=u_{e m}(t)$ and $\eta_{m}^{s}(t)+c_{e}(t)<\eta_{l}^{s}(t)$, then $\delta_{\mathrm{e}}(t)>0$ and $\delta_{\mathrm{e}}(t)=\eta_{1}^{\mathrm{s}}(t)-\eta_{m}^{\mathrm{s}}(t)-c_{\mathrm{e}}(t)$.

The optimal solutions which are subject to (8) (13) are denoted as $u_{a}^{s^{*}}(t), u_{e}^{s^{*}}(t)$.

\subsection{VI model equivalent to DUO condition based on instantaneous travel time}

The time range $[0, T]$ is discretized into many equally small time intervals $0,1,2, \ldots, K$. For time interval $k$, actual travel time $\tau_{a}(k)$ on link $a$ is temporally fixed and rounded to the nearest integer.

1) Phase equations

$$
\begin{aligned}
& x_{a}^{s}(k)=x_{a}^{s}(k-1)+u_{a}^{s}(k-1)-v_{a}^{s}(k-1), \\
& \forall a, s ; k=1,2, \cdots, k
\end{aligned}
$$


$\hat{x}_{p}^{s}(k)=\hat{x}_{p}^{s}(k-1)+\hat{u}_{p}^{s}(k-1)-\hat{v}_{p}^{s}(k-1)$,

$\forall p, s ; k=1,2, \cdots, k$

2) Flow conservation constraints at nodes For node I in the road network, there is

$\sum_{a \in E_{l}} v_{a}^{s}(k)+g_{l}^{s}(k)=\sum_{a \in F_{l}} u_{a}^{s}(k), \quad \forall I, s, k$

At the joint of the park $p$ and its access passage e, there is

$\hat{u}_{p}^{s}(k)=v_{\mathrm{e}}^{\mathrm{s}}(k)$

3) Flow propagation constraints

For road link $a$ and time interval $k, l_{a, k}$ is a set whose elements $\left(i_{a, k}\right)$ satisfy $i_{a, k}+\tau_{a}\left(i_{a, k}\right)=k$. There is

$\sum_{i_{a, k} \in I_{a, k}} u_{a}^{s}\left(i_{a, k}\right)=v_{a}^{s}(k), \forall a, s, k$

For park $p$, there is

$\sum_{i_{p, k}^{s}, i_{p, k}^{s}} \hat{u}_{p}^{s}\left(\hat{i}_{p, k}^{s}\right)=\hat{v}_{p}^{s}(k), \forall p, s, k$

For passage e, the number of vehicles entering the park from the passage in time interval $k$ is

$v_{e}(k)=\min \left(\min \left(x_{e}(k)-\left\{u_{e}(k-1)+u_{e}(k-2)+\right.\right.\right.$

$\left.\left.\left.+\cdots+u_{e}\left[k-\left(D_{e}-1\right)\right]\right\}, \frac{C_{e}}{D_{e}}\right), Q_{p}-\hat{x}_{p}(k)+\hat{v}_{p}(k)\right)$

where $e$ is the access passage of park $p . v_{e}^{s}(k)$ was analysed in Figure 8.

4) Capacity constraint of the passage

$u_{e}(k) \leq \min \left(\frac{C_{e}}{D_{e}}, C_{e}-x_{e}(k)+v_{e}(k)\right), \forall e, s, k$

5) Non-negative constraint

$x_{a}^{s}(k) \geq 0, u_{a}^{s}(k) \geq 0, v_{a}^{s}(k) \geq 0, \quad \forall a, s, k$

$\hat{X}_{p}^{s}(k) \geq 0, \hat{u}_{p}^{s}(k) \geq 0, \hat{v}_{p}^{s}(k) \geq 0, \quad \forall p, s, k$

6) Boundary (initial) condition

$x_{a}^{s}(0)=0, v_{a}^{s}(0)=0, \quad \forall a, s$

$\hat{X}_{p}^{s}(0)=0, \hat{v}_{p}^{s}(0)=0, \quad \forall p, s$

The set of feasible dynamic flows satisfying (14) (29) is denoted as $\Omega$.

VI problem equivalent to DUO condition based on instantaneous travel time can be written as theorem 3 .

Theorem 3

Dynamic feasible flow set $\Omega$ is under the instantaneous DUO condition if and only if they satisfy the $\mathrm{VI}$ problem (VIP1).

(VIP1)

$\sum_{s} \sum_{a}\left[\eta_{m}^{s^{*}}(t)+c_{a}^{*}(t)\right]\left[u_{a}^{s}(t)-u_{a}^{s^{*}}(t)\right] \geq 0$,

$\forall u_{a}^{s}(t) \in \Omega$

The proof of equivalence of $\mathrm{VI}(26)$ and the DUO condition can be seen in Gao and Ren [16].
It is known from the constraints that, $x_{a}^{s}(k), x_{p}^{s}(k)$ can be obtained from (14), (15) according to the data of time interval $k-1$, and $v_{a}^{s}(k), \hat{v}_{p}^{s}(k), v_{e}^{s}(k)$ can be obtained from (18), (19), (20) according to the data of previous time intervals. So, in time interval $k, x_{a}^{s}(k)$, $\hat{x}_{p}^{s}(k), v_{a}^{s}(k), v_{e}^{s}(k)$ (i.e. $\left.\hat{u}_{p}^{s}(k)\right), \hat{v}_{p}^{s}(k)$ are known and the only variables are $u_{a}^{s}(k)$ (including $u_{e}^{s}(k)$ ). After the time range $[0, T]$ is discretized into $K+1$ equally small time intervals $0,1,2, \ldots, K,(26)$ is converted into (VIP2).

(VIP2)

$\sum_{s} \sum_{a}\left[\eta_{m}^{s^{*}}(k)+c_{a}^{*}(k)\right]\left[u_{a}^{s}(k)-u_{a}^{s^{*}}(k)\right] \geq 0$,

$\forall k=0,1,2, \cdots, k$

subject to

$\sum_{a \in E_{l}} \sum_{i_{a}, k} \in I_{a, k} v_{a}^{s}\left(i_{a, k}\right)+g_{l}^{s}(k)=\sum_{a \in F_{l}} u_{a}^{s}(k), \quad \forall I, s, k$

$u_{e}(k) \leq \min \left(\frac{C_{e}}{D_{e}}, C_{e}-x_{e}(k)+v_{e}(k)\right), \forall e, s, k$

$u_{a}^{s}(k) \geq 0, \forall a, s, k$

\section{SOLUTION ALGORITHM}

In Diagonalization (relaxation) algorithm, the solution of VI problem (VIP2) is decomposed into a series of solutions of sub-problems. In the solution of each sub-problem, $\eta_{m}^{s}(k)$ is temporarily fixed as $\bar{\eta}_{m}^{s}(k)$. The sub-problem is (VIP3).

(VIP3)

$\sum_{s} \sum_{a}\left[\bar{\eta}_{m}^{s^{*}}(k)+c_{a}^{*}(k)\right]\left[u_{a}^{s}(k)-u_{a}^{s^{*}}(k)\right] \geq 0$,

$\forall k=0,1,2, \cdots, k$

subject to (28) (30).

Each sub-problem corresponds to an equivalent non-linear programming problem (NLP1).

(NLP1)

$\min _{\mathbf{u}^{s}} Z[\mathbf{u}(k)]=\sum_{a} \int_{0}^{u_{a}(k)} c_{a}(\omega) d \omega+\sum_{s} \sum_{a} \bar{\eta}_{m}^{s}(k) u_{a}^{s}(k)$,

$\forall k=0,1,2, \ldots, k$

s.t (28) (30).

where $\mathbf{u}^{\mathrm{s}}(k)$ is a vector whose elements are $u_{a}^{s}(k)$, $\mathbf{u}(k)$ is a vector whose elements are $u_{a}(k)$. To prove the equivalence of (NLP1) and (VIP3) one only needs to prove that the gradient of (32) equals the cost item of (31).

$\frac{\partial Z(\mathbf{u})}{\partial u_{a}^{s}(k)}=c_{a}(k)+\bar{\eta}_{m}^{s}(k), \quad \forall a, s, k$

The Frank-Wolfe algorithm [17] is applied to solve (NLP1). In each iteration, solve a linear programming sub-problem (34)-(37).

$\min _{\mathrm{h}^{\mathrm{s}}(k)} \hat{Z}=\nabla_{\mathbf{u}^{\mathrm{s}}(k)} Z[\mathbf{u}(k)] \mathrm{h}^{\mathrm{s}^{\mathrm{T}}}(k), \forall k$ 
s.t

$\sum_{a \in E_{l}} \sum_{i_{a}, k I_{a, k}} v_{a}^{s}\left(i_{a, k}\right)+g_{l}^{s}(k)=\sum_{a \in F_{l}} h_{a}^{s}(k), \quad \forall l \neq p, s ; s, k$

$h_{e}(k) \leq \min \left(\frac{C_{e}}{D_{e}}, C_{e}-x_{e}(k)+v_{e}(k)\right), \forall e, s, k$

$h_{a}^{s}(k) \geq 0, \quad \forall a, s, k$

where $\mathbf{h}^{\mathrm{s}}(k)$ is the feasible solution of the sub-problem (34)-(37) corresponding to $\mathbf{u}^{\mathrm{s}}(k)$.

In the shortest path algorithm to find a feasible solution of $\mathbf{h}^{\mathrm{s}}(k)$, the travel time on the road link is

$t_{a}^{s}(k)=\frac{\partial Z(u)}{\partial u_{a}^{s}(k)}=c_{a}\left[u_{a}(k)\right]+\bar{\eta}_{m}^{s}(k), \forall a, s, k$

Let us analyse the influence of capacity constraint of the passage on flow assignment. In Figure 9, assume the flow at node 3 to enter park 5 has three destinations, namely $6,7,8$. Links $3 \rightarrow 2$ and $3 \rightarrow 5$ both belong to $F_{1}(l=3)$, and $3 \rightarrow 5$ is the access passage of the park.

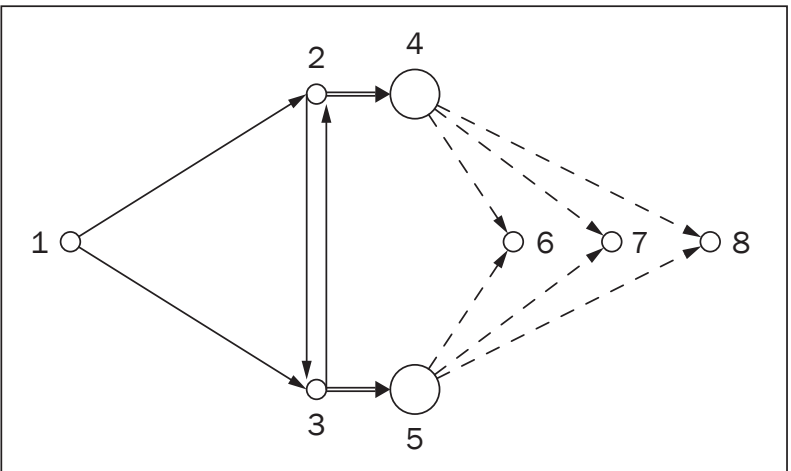

Figure 9 - Analysis under capacity constraint of the passage

In the Frank-Wolfe algorithm, all vehicles are assigned to the shortest path of $(I, s)$ without capacity constraint of the passage. However, because of the capacity constraint of the passage, the shortest path to destination $s$ not through $e \in F$, at node 3 needs to be found. Here, capacity constraints of road links are ignored. Suppose that at the gate $(I=3)$ of the passage, the shortest paths to destinations $6,7,8$ are $L_{1}$, $L_{2}, L_{3}$, and $L_{1}, L_{2}$, respectively, pass through the passage $e \in F_{1}$ but $L_{3}$ does not pass through $e \in F_{1}$. The shortest paths to destinations 6,7 not through $e \in F_{\text {, }}$ are $L_{4}, L_{5}$, respectively. $h_{a}^{s}(k)$ can be obtained from solving (46) (60).

$\min \left(R_{L_{1}} y_{1}+R_{L_{4}} y_{2}+R_{L_{2}} y_{3}+R_{L_{5}} y_{4}+R_{L_{3}} y_{5}\right)$

s.t

$\sum_{a \in F_{l}} h_{a}^{s}(k)=\sum_{a \in E_{l}} \sum_{i_{a, k} \in l_{a, k}} v_{a}^{s}\left(i_{a, k}\right)+g_{l}^{s}(k)=G_{l}^{s}(k)$,

$\forall I \neq p, s ; s, k$

$y_{1}+y_{2}=G_{3}^{6}(k)$
$y_{3}+y_{4}=G_{3}^{7}(k)$

$y_{5}=h_{3 \rightarrow 2}^{8}(k)=G_{3}^{8}(k)$

$y_{1}=h_{3 \rightarrow 5}^{6}(k)$

$y_{2}=h_{3 \rightarrow 2}^{6}(k)$

$y_{3}=h_{3 \rightarrow 5}^{7}(k)$

$y_{4}=h_{3 \rightarrow 2}^{7}(k)$

$R_{L_{1}}=c_{3 \rightarrow 5}\left[u_{3 \rightarrow 5}(k)\right]+\bar{\eta}_{5}^{6}(k)$

$R_{L_{2}}=c_{3 \rightarrow 5}\left[u_{3 \rightarrow 5}(k)\right]+\bar{\eta}_{5}^{7}(k)$

$R_{L_{3}}=c_{3 \rightarrow 2}\left[u_{3 \rightarrow 2}(k)\right]+\bar{\eta}_{2}^{8}(k)$

$R_{L_{4}}=c_{3 \rightarrow 2}\left[u_{3 \rightarrow 2}(k)\right]+\bar{\eta}_{2}^{6}(k)$

$R_{L_{5}}=c_{3 \rightarrow 2}\left[u_{3 \rightarrow 2}(k)\right]+\bar{\eta}_{2}^{7}(k)$

and, the flows on paths $L_{1}, L_{2}$, which are through $e \in F_{1}$, satisfy

$y_{1}+y_{3} \leq \min \left(\frac{C_{3 \rightarrow 5}}{D_{3 \rightarrow 5}}, C_{3 \rightarrow 5}-x_{3 \rightarrow 5}(k)+v_{3 \rightarrow 5}(k)\right)$

Solve (39) (53) to calculate $y_{1}, y_{2}, y_{3}, y_{4}, y_{5}$ and then $h_{a}^{s}(k), a \in F_{3}$.

Steps of Diagonalization (relaxation) algorithm are: Step 0 - For time interval $k$, the actual travel time on link a $(\forall a)$ is rounded to an integer $\left(\tau_{a}^{0}(k)\right)$ and the value of $\tau_{a}^{0}(k)(\forall a)$ has been supposed. Calculate set $I_{a, k}^{0}$. Let $N=0$.

Step 1 - Initial conditions: $x_{a}^{s}(0)=0, v_{a}^{s}(0)=0(\forall a, s)$, $\hat{x}_{p}^{s}(0)=0, \hat{v}_{p}^{s}(0)=0(\forall p, s)$. Let $k=0$.

Step 2 - According to link-free flow travel time, carry out all-or-nothing assignment (if it is at the entrance node of access passage of the park, then calculate (39) (53)) of flows $\sum_{a \in E_{l}} \sum_{i_{a} \in \epsilon_{a}^{N},} v_{a}^{s^{N}}\left(i_{a, k}\right)+g_{l}^{s}(k), \forall I \neq p, s ; s, k$

( $g_{1}^{s}(0), \forall I \neq p, s ; s, k$ if $k=0$ ) to obtain initial rate of inflow $\left(u_{a}^{(0)}(k)\right)$ of links. Let $n=1$.

Step 3 - Calculate instantaneous travel time on each link $c_{a}^{(n-1)}(k)=c_{a}\left[x_{a}^{N}(k), u_{a}^{(n-1)}(k), v_{a}^{N}(k)\right]+\bar{\eta}_{m}^{s^{N-1}}(k)$,

where $\bar{\eta}_{m}^{s^{N-1}}(k)=\bar{\eta}_{m}^{s^{N-1}}\left[\mathbf{x}^{N}(k), \mathbf{u}^{N-1}(k), \mathbf{v}^{N}(k)\right]$ is a temporarily fixed value of the shortest instantaneous path travel time from $m$ to $s$. Carry out all-or-nothing assignment (if it is at the entrance node of access passage of the park, then calculate (39) (53)) of flows $\sum_{a \in E_{1}} \sum_{i_{2} \in l^{N}} v_{a}^{s^{N}}\left(i_{a, k}\right)+g_{l}^{s}(k), \forall I \neq p, s ; s, k$

( $g_{1}^{s}(0), \forall I \neq p, s ; s, k$ if $k=0$ ) to obtain feasible rate of in-flow of links $\left\{h_{a}^{s^{(n-1)}}(k)\right\}$ and then $\left\{h_{a}^{(n-1)}(k)\right\}$. 
Step 4 - One-dimensional search. Solve

$\min _{0 \leq \gamma \leq 1} Z=\sum_{a} \int_{0}^{u_{a}^{(n-1)}(k)+\gamma\left[h_{a}^{(n-1)}(k)-u_{a}^{(n-1)}(k)\right]} c_{a}(\omega) d \omega+$

$+\sum_{a} \sum_{s} \bar{\eta}_{m}^{s^{N-1}}(k)\left\{u_{a}^{s^{(n-1)}}(k)+\gamma\left[h_{a}^{s^{(n-1)}}(k)-u_{a}^{s^{(n-1)}}(k)\right]\right\}$

to obtain step length $\gamma^{(n-1)}$.

Step 5 - Flows update: let

$u_{a}^{s^{(n)}}(k)=u_{a}^{s^{(n-1)}}(k)+\gamma^{(n-1)}\left[h_{a}^{s^{(n-1)}}(k)-u_{a}^{s^{(n-1)}}(k)\right],(\forall a, s)$

and then calculate $\left\{u_{a}^{(n)}(k)\right.$.

Step 6 - If $n=n_{m}$ ( $n_{m}$ is the set maximal iterative number for the inner loop), then turn to step 7; while if $n<n_{m}$, then let $n=n+1$ and turn to step 3 .

Step 7 - If $k=K$, then turn to step 8; else, set $k=k+1$ and turn to step 2.

Step 8 - Convergence check. Estimate actual travel time on links $\tau_{a}^{N}(k)(\forall k)$. If $\tau_{a}^{N}(k) \approx \tau_{a}^{N-1}(k)$, then $u_{a}(k)=u_{a}^{(n)^{N}}(k)(\forall a, k)$, where $u_{a}^{(n)^{N}}(k)$ is the value of $u_{a}^{(n)}(k)$ at the $N^{\text {th }}$ iteration of outer loop, end; else, $u_{a}^{N}(k)=u_{a}^{(n)^{N}}(k)$, calculate $l_{a, k}^{N}$, let $N=N+1$ and turn to step 1.

The number of iterations of the outer loop required relates to that of the inner layer. If the number of iterations of the outer loop is large, that of the inner loop can be small, meaning that Frank-Wolfe algorithm of the inner loop (steps 3 6) does not have to be convergent. This is called simplified diagonalization algorithm, and the whole convergence is good when the number of iterations of the inner loop takes 2 3 [18]. The algorithm (steps 0 8) ends when the actual travel time does not change or the change is small [8].

\section{NUMERICAL EXAMPLE}

In Figure 3, the total number of time intervals $K=20$, and parking durations of vehicles entering parks (nodes 4 and 5 ) at each time point are shown in Table 1. O-D (origin-destination) traffic demands are in Table 2. The number of parking spaces in parks: $Q_{4}=10, Q_{5}=10$. The time for walking egress trip from parks to the destination: $B_{4,6}=1, B_{5,6}=1$. The maximal number of queuing vehicles that the access passage of parks can accommodate at the same time: $C_{2 \rightarrow 4}=5$, $C_{3 \rightarrow 5}=5$.
Instantaneous travel time on links:

$c_{a}(t)=0.5+c_{a, 0}+0.001\left(u_{a}(t)\right)^{2}+0.001\left(v_{a}(t)\right)^{2}+$ $+0.005\left(x_{a}(t)\right)^{2}, \quad \forall a, t$, where $c_{a, 0}$ is the free flow travel time on link $a$, and $c_{1 \rightarrow 2,0}=3, c_{1 \rightarrow 3,0}=3.5$, $c_{2 \rightarrow 3,0}=0.5, c_{3 \rightarrow 2,0}=0.5$. The initial actual travel times on links are assumed as: $\tau_{1 \rightarrow 2}(k)=2, \tau_{1 \rightarrow 3}(k)=2$, $\tau_{2 \rightarrow 3}(k)=2, \tau_{3 \rightarrow 2}(k)=2, k=0,1,2, \ldots, 19$. Here, suppose $\tau_{a}(t)=\operatorname{round}\left(c_{a}(t)\right) . D_{2 \rightarrow 4}=1, D_{3 \rightarrow 5}=1$, then (5) is simplified as $v_{e}(k)=\min \left(x_{e}(k), Q_{p}-x_{p}(k)+v_{p}(k)\right)$. Assume instantaneous travel time on access passages of parks without parking information available:

If $v_{2 \rightarrow 4}(k)>0$, then $c_{2 \rightarrow 4}(k)=\max \left(1, \operatorname{round}\left(\frac{x_{2 \rightarrow 4}(k)}{v_{2 \rightarrow 4}(k)}\right)\right)$;

If $v_{3 \rightarrow 5}(k)>0$, then $c_{3 \rightarrow 5}(k)=1+\operatorname{round}\left(\frac{x_{3 \rightarrow 5}(k)}{v_{3 \rightarrow 5}(k)}\right)$;

and when vehicles queuing occurs in the passage but $v_{e}(k)=0, c_{e}(k)$ takes a very large value, which is supposed to be infinity; while when $v_{e}(k)=0$ but no vehicles queuing occurs in the passage, $c_{e}(k)=0$.

The precision takes 0.01 , and the solutions from diagonalization algorithm are in Table 3 . When $k$ is time intervals $0 \sim 4,8,15,17,18$ or $19, v_{2 \rightarrow 4}(k)=0$, $x_{2 \rightarrow 4}(k)=0$ which means no vehicles are queuing in the passage, so $c_{2 \rightarrow 4}(k)=1$ according to the supposition. When $k$ is time intervals $5,6,7,9,10,12,14$ or 16 , $c_{2 \rightarrow 4}(k)=\max \left(1, \operatorname{round}\left(\frac{x_{2 \rightarrow 4}(k)}{v_{2 \rightarrow 4}(k)}\right)\right)=1$. When $k$ is time interval 11, $c_{2 \rightarrow 4}(k)=\max \left(1, \operatorname{round}\left(\frac{x_{2 \rightarrow 4}(k)}{v_{2 \rightarrow 4}(k)}\right)\right)=2$. When $k$ is time interval 13, $x_{2 \rightarrow 4}(k)=1.5625$ but $v_{2 \rightarrow 4}(k)=0$, so $c_{2 \rightarrow 4}(k)$ takes a large value. When $k$ is time intervals $0 \sim 6,10,12,14$ or $16 \sim 19, v_{3 \rightarrow 5}(k)=0, x_{3 \rightarrow 5}(k)=0$ which means no vehicles are queuing in the passage, so $c_{3 \rightarrow 5}(k)=1$. When $k$ is time intervals $7,8,9,11,13$ or $15, c_{3 \rightarrow 5}(k)=1+\operatorname{round}\left(\frac{x_{3 \rightarrow 5}(k)}{v_{3 \rightarrow 5}(k)}\right)=2$.

In time interval 5, 6 units of flows come out of link $1 \rightarrow 2$, meanwhile 4 units of flows just go from passage $2 \rightarrow 4$ to the park. There have been 4 units of flows in the passage $2 \rightarrow 4$ by the end of time interval 4 , so 5 units of flows can go into the passage $2 \rightarrow 4$ in time interval 5 , and the remaining 1 unit of flows go from node 2 to

Table 1 - Parking durations of vehicles entering parks at each time point

\begin{tabular}{|c|c|c|c|c|c|c|c|c|c|c|c|c|c|c|c|c|c|c|c|c|}
\hline \multirow{2}{*}{$\begin{array}{c}\text { Parking } \\
\text { durations }\end{array}$} & \multicolumn{20}{|c|}{ Time points $(k)$} \\
\hline & 0 & 1 & 2 & 3 & 4 & 5 & 6 & 7 & 8 & 9 & 10 & 11 & 12 & 13 & 14 & 15 & 16 & 17 & 18 & 19 \\
\hline$T_{4}(k)$ & 3 & 4 & 4 & 5 & 3 & 2 & 2 & 4 & 4 & 3 & 4 & 4 & 2 & 3 & 2 & 3 & 1 & 1 & 4 & 1 \\
\hline$T_{5}(k)$ & 2 & 1 & 5 & 4 & 3 & 4 & 5 & 3 & 2 & 5 & 2 & 4 & 2 & 4 & 3 & 1 & 2 & 3 & 1 & 3 \\
\hline
\end{tabular}

Table 2 - O-D traffic demands

\begin{tabular}{|c|c|c|c|c|c|c|c|c|c|c|c|c|c|c|c|c|c|c|c|c|}
\hline \multirow{2}{*}{$O-D$} & \multicolumn{20}{|c|}{ Time intervals $(k)$} \\
\hline & 0 & 1 & 2 & 3 & 4 & 5 & 6 & 7 & 8 & 9 & 10 & 11 & 12 & 13 & 14 & 15 & 16 & 17 & 18 & 19 \\
\hline $1 \rightarrow 6$ & 4 & 6 & 4 & 5 & 5 & 6 & 4 & 6 & 4 & 5 & 0 & 0 & 0 & 0 & 0 & 0 & 0 & 0 & 0 & 0 \\
\hline
\end{tabular}


node 3 and then go into the passage $3 \rightarrow 5$ in one time interval and finally park at the park node 5 to walk to destination node 6 . At time point 7 , the amount of flows in the passage $3 \rightarrow 5$ is 3.0020 , which is composed of 2.0020 coming out of link $1 \rightarrow 3$ and 1 coming out of link $2 \rightarrow 3$ in time interval 6 . In time interval 9, 6 units of flows come out of link $1 \rightarrow 2$, meanwhile 3.4375 units of flows just go from passage $2 \rightarrow 4$ to the park. There have been 3.4375 units of flows in the passage $2 \rightarrow 4$ by the end of time interval 8, so 5 units of flows can go into the

Table 3 - Solutions of test network passage $2 \rightarrow 4$ in time interval 9 , and the remaining 1 unit of flows go from node 2 to node 3 and then go into the passage $3 \rightarrow 5$ in one time interval and finally park at the park node 5 to walk to destination node 6 .

In time interval 11 , rate of out-flow of link $1 \rightarrow 2$ is 6 , in which 2.5625 units of flows go into the passage $2 \rightarrow 4$, and the remaining 3.4375 units of flows go from node 2 to node 3 and then arrive at node 3 in one time interval (namely in time interval 12). But at node 3 , not all flows go to the park. The accumulated flows

\begin{tabular}{|c|c|c|c|c|c|c|c|c|c|c|c|}
\hline 足 & 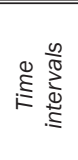 & 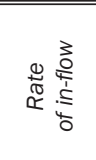 & 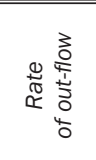 & $\frac{\sum_{0}^{\infty}}{\mathbb{L}}$ & 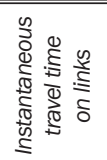 & $\begin{array}{ll}\mathscr{0} & \\
0 & 0 \\
0 & = \\
0 & \pi \\
0 & 0 \\
0 & 0 \\
0 & 0\end{array}$ & 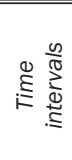 & 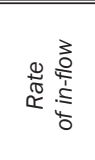 & 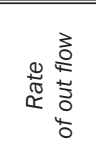 & $\frac{0}{0}$ & 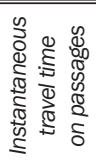 \\
\hline \multirow{20}{*}{$1 \rightarrow 2$} & 0 & 4 & 0 & 0 & 3.5160 & \multirow{20}{*}{$2 \rightarrow 4$} & 0 & 0 & 0 & 0 & 1 \\
\hline & 1 & 6 & 0 & 4 & 3.6160 & & 1 & 0 & 0 & 0 & 1 \\
\hline & 2 & 1.9980 & 0 & 10 & 4.0040 & & 2 & 0 & 0 & 0 & 1 \\
\hline & 3 & 0 & 0 & 11.9980 & 4.2198 & & 3 & 0 & 0 & 0 & 1 \\
\hline & 4 & 3.4351 & 4 & 11.9980 & 4.2476 & & 4 & 4 & 0 & 0 & 1 \\
\hline & 5 & 6 & 6 & 11.4331 & 4.2256 & & 5 & 5 & 4 & 4 & 1 \\
\hline & 6 & 4.0000 & 1.9980 & 11.4331 & 4.1736 & & 6 & 1.9980 & 5 & 5 & 1 \\
\hline & 7 & 6.0000 & 0 & 13.4351 & 4.4385 & & 7 & 0 & 1.9980 & 1.9980 & 1 \\
\hline & 8 & 0 & 3.4351 & 19.4351 & 5.4004 & & 8 & 3.4375 & 0 & 0 & 1 \\
\hline & 9 & 5 & 6 & 16 & 4.8410 & & 9 & 5 & 3.4375 & 3.4375 & 1 \\
\hline & 10 & 0 & 4 & 15 & 4.6410 & & 10 & 4 & 4.5645 & 5 & 1 \\
\hline & 11 & 0 & 6 & 11 & 4.1410 & & 11 & 2.5625 & 1.9980 & 4.4355 & 2 \\
\hline & 12 & 0 & 0 & 5 & 3.6250 & & 12 & 0 & 3.4375 & 5 & 1 \\
\hline & 13 & 0 & 5 & 5 & 3.6500 & & 13 & 0 & 0 & 1.5625 & infinity \\
\hline & 14 & 0 & 0 & 0 & 3.5000 & & 14 & 0 & 1.5625 & 1.5625 & 1 \\
\hline & 15 & 0 & 0 & 0 & 3.5000 & & 15 & 2.4375 & 0 & 0 & 1 \\
\hline & 16 & 0 & 0 & 0 & 3.5000 & & 16 & 0 & 2.4375 & 2.4375 & 1 \\
\hline & 17 & 0 & 0 & 0 & 3.5000 & & 17 & 0 & 0 & 0 & 1 \\
\hline & 18 & 0 & 0 & 0 & 3.5000 & & 18 & 0 & 0 & 0 & 1 \\
\hline & 19 & 0 & 0 & 0 & 3.5000 & & 19 & 0 & 0 & 0 & 1 \\
\hline \multirow{20}{*}{$1 \rightarrow 3$} & 0 & 0 & 0 & 0 & 4.0000 & \multirow{20}{*}{$3 \rightarrow 5$} & 0 & 0 & 0 & 0 & 1 \\
\hline & 1 & 0 & 0 & 0 & 4.0000 & & 1 & 0 & 0 & 0 & 1 \\
\hline & 2 & 2.0020 & 0 & 0 & 4.0040 & & 2 & 0 & 0 & 0 & 1 \\
\hline & 3 & 5 & 0 & 2.0020 & 4.0450 & & 3 & 0 & 0 & 0 & 1 \\
\hline & 4 & 1.5649 & 0 & 7.0020 & 4.2476 & & 4 & 0 & 0 & 0 & 1 \\
\hline & 5 & 0 & 0 & 8.5669 & 4.3670 & & 5 & 0 & 0 & 0 & 1 \\
\hline & 6 & 0 & 2.0020 & 8.5669 & 4.3710 & & 6 & 3.0020 & 0 & 0 & 1 \\
\hline & 7 & 0 & 5 & 6.5649 & 4.2405 & & 7 & 4.9976 & 3.0020 & 3.0020 & 2 \\
\hline & 8 & 4.0000 & 1.5649 & 1.5649 & 4.0307 & & 8 & 1.5649 & 4.9976 & 4.9976 & 2 \\
\hline & 9 & 0 & 0 & 4 & 4.0800 & & 9 & 0 & 1.5649 & 1.5649 & 2 \\
\hline & 10 & 0 & 0 & 4 & 4.0800 & & 10 & 1 & 0 & 0 & 1 \\
\hline & 11 & 0 & 0 & 4 & 4.0800 & & 11 & 0 & 1 & 1 & 2 \\
\hline & 12 & 0 & 4 & 4 & 4.0960 & & 12 & 5 & 0 & 0 & 1 \\
\hline & 13 & 0 & 0 & 0 & 4.0000 & & 13 & 0 & 5 & 5 & 2 \\
\hline & 14 & 0 & 0 & 0 & 4.0000 & & 14 & 5 & 0 & 0 & 1 \\
\hline & 15 & 0 & 0 & 0 & 4.0000 & & 15 & 0 & 5 & 5 & 2 \\
\hline & 16 & 0 & 0 & 0 & 4.0000 & & 16 & 0 & 0 & 0 & 1 \\
\hline & 17 & 0 & 0 & 0 & 4.0000 & & 17 & 0 & 0 & 0 & 1 \\
\hline & 18 & 0 & 0 & 0 & 4.0000 & & 18 & 0 & 0 & 0 & 1 \\
\hline & 19 & 0 & 0 & 0 & 4.0000 & & 19 & 0 & 0 & 0 & 1 \\
\hline
\end{tabular}


(7.4375) at node 3 in time interval 12 is composed of 4 from link $1 \rightarrow 3$ and 3.4375 from link $2 \rightarrow 3$, in which 5 units of flows go into the passage $3 \rightarrow 5$ and the remaining 2.4375 go back to node 2 (namely $3 \rightarrow 2$ ), so cruising traffic appears here. The accumulated flows (7.4375) at node 2 in time interval 13 is composed of back flows (2.4375) just from $3 \rightarrow 2$ and 5 units of flows coming out of link $1 \rightarrow 2$. Here, vehicles queue in the access passage of the park (park node 4) but rate of out-flow of the passage is 0 , so $c_{e}(k)$ takes a large

Table 3 (continued) - Solutions of test network

\begin{tabular}{|c|c|c|c|c|c|c|c|c|c|c|c|}
\hline$\stackrel{\text { N }}{\stackrel{5}{\Xi}}$ & 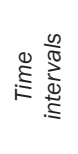 & 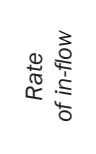 & 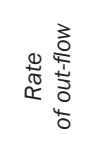 & $\frac{\sum_{0}^{\infty}}{4}$ & 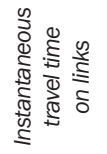 & \begin{tabular}{ll}
0 & 0 \\
0 & 0 \\
$\pi$ & \multirow{2}{*}{} \\
0 & $\mathbb{2}$ \\
0 & 0 \\
$\pi$ & 0 \\
0 & 0
\end{tabular} & 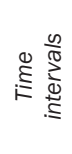 & $\underset{0}{\frac{0}{0}}$ & 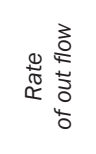 & $\frac{\sum_{0}^{\infty}}{4}$ & 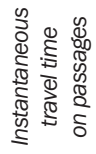 \\
\hline \multirow{20}{*}{$2 \rightarrow 3$} & 0 & 0 & 0 & 0 & 1.0000 & \multirow{20}{*}{4} & 0 & 0 & 0 & 0 & - \\
\hline & 1 & 0 & 0 & 0 & 1.0000 & & 1 & 0 & 0 & 0 & - \\
\hline & 2 & 0 & 0 & 0 & 1.0000 & & 2 & 0 & 0 & 0 & - \\
\hline & 3 & 0 & 0 & 0 & 1.0000 & & 3 & 0 & 0 & 0 & - \\
\hline & 4 & 0 & 0 & 0 & 1.0000 & & 4 & 0 & 0 & 0 & - \\
\hline & 5 & 1 & 0 & 0 & 1.0010 & & 5 & 4 & 0 & 0 & - \\
\hline & 6 & 0 & 1 & 1 & 1.0060 & & 6 & 5 & 0 & 4 & - \\
\hline & 7 & 0 & 0 & 0 & 1.0000 & & 7 & 1.9980 & 4 & 9 & - \\
\hline & 8 & 0 & 0 & 0 & 1.0000 & & 8 & 0 & 5 & 6.9980 & - \\
\hline & 9 & 1 & 0 & 0 & 1.0010 & & 9 & 3.4375 & 0 & 1.9980 & - \\
\hline & 10 & 0 & 1 & 1 & 1.0060 & & 10 & 4.5645 & 0 & 5.4355 & - \\
\hline & 11 & 3.4375 & 0 & 0 & 1.0118 & & 11 & 1.9980 & 1.9980 & 10 & - \\
\hline & 12 & 0 & 3.4375 & 3.4375 & 1.0709 & & 12 & 3.4375 & 3.4375 & 10 & - \\
\hline & 13 & 7.4375 & 0 & 0 & 1.0553 & & 13 & 0 & 0 & 10 & - \\
\hline & 14 & 0 & 7.4375 & 7.4375 & 1.3319 & & 14 & 1.5625 & 8.0020 & 10 & - \\
\hline & 15 & 0 & 0 & 0 & 1.0000 & & 15 & 0 & 1.9980 & 3.5605 & - \\
\hline & 16 & 0 & 0 & 0 & 1.0000 & & 16 & 2.4375 & 1.5625 & 1.5625 & - \\
\hline & 17 & 0 & 0 & 0 & 1.0000 & & 17 & 0 & 2.4375 & 2.4375 & - \\
\hline & 18 & 0 & 0 & 0 & 1.0000 & & 18 & 0 & 0 & 0 & - \\
\hline & 19 & 0 & 0 & 0 & 1.0000 & & 19 & 0 & 0 & 0 & - \\
\hline \multirow{20}{*}{$3 \rightarrow 2$} & 0 & 0 & 0 & 0 & 1.0000 & \multirow{20}{*}{5} & 0 & 0 & 0 & 0 & - \\
\hline & 1 & 0 & 0 & 0 & 1.0000 & & 1 & 0 & 0 & 0 & - \\
\hline & 2 & 0 & 0 & 0 & 1.0000 & & 2 & 0 & 0 & 0 & - \\
\hline & 3 & 0 & 0 & 0 & 1.0000 & & 3 & 0 & 0 & 0 & - \\
\hline & 4 & 0 & 0 & 0 & 1.0000 & & 4 & 0 & 0 & 0 & - \\
\hline & 5 & 0 & 0 & 0 & 1.0000 & & 5 & 0 & 0 & 0 & - \\
\hline & 6 & 0 & 0 & 0 & 1.0000 & & 6 & 0 & 0 & 0 & - \\
\hline & 7 & 0.0024 & 0 & 0 & 1.0000 & & 7 & 3.0020 & 0 & 0 & - \\
\hline & 8 & 0 & 0.0024 & 0.0024 & 1.0000 & & 8 & 4.9976 & 0 & 3.0020 & - \\
\hline & 9 & 0 & 0 & 0 & 1.0000 & & 9 & 1.5649 & 0 & 7.9995 & - \\
\hline & 10 & 0 & 0 & 0 & 1.0000 & & 10 & 0 & 7.9995 & 9.5645 & - \\
\hline & 11 & 0 & 0 & 0 & 1.0000 & & 11 & 1 & 0 & 1.5649 & - \\
\hline & 12 & 2.4375 & 0 & 0 & 1.0059 & & 12 & 0 & 0 & 2.5649 & - \\
\hline & 13 & 0 & 2.4375 & 2.4375 & 1.0356 & & 13 & 5 & 0 & 2.5649 & - \\
\hline & 14 & 2.4375 & 0 & 0 & 1.0059 & & 14 & 0 & 1.5649 & 7.5649 & - \\
\hline & 15 & 0 & 2.4375 & 2.4375 & 1.0356 & & 15 & 5 & 1 & 6 & - \\
\hline & 16 & 0 & 0 & 0 & 1.0000 & & 16 & 0 & 5 & 10 & - \\
\hline & 17 & 0 & 0 & 0 & 1.0000 & & 17 & 0 & 5 & 5 & - \\
\hline & 18 & 0 & 0 & 0 & 1.0000 & & 18 & 0 & 0 & 0 & - \\
\hline & 19 & 0 & 0 & 0 & 1.0000 & & 19 & 0 & 0 & 0 & - \\
\hline
\end{tabular}

value according to the supposition, and then these 7.4375 units of flows go from node 2 to node 3 and arrive at node 3 in one time interval (namely in time interval 14). But at node 3 , not all flows go to the park. In time interval 14, 5 of 7.4375 units of flows go into the passage and the remaining 2.4375 go back to node 2 , so here cruising traffic appears. These 2.4375 units of flows arrive at node 2 in one time interval (namely in time interval 15) and all go into passage $2 \rightarrow 4$, and finally park at the park node 4 to walk to destination 6 . 


\section{CONCLUSIONS AND PROSPECTS}

This paper has presented a dynamic parking choice model to simulate drivers' search for parking spaces between parking lots and then to obtain the data of vehicles going into or out of the park timely, traffic flows on the surrounding road network, and the amount and routes of cruising traffic owing to parking search. Modelling dynamic process of parking search helps evaluate whether the number and layout of parking lots is able to satisfy dynamic parking demands, so a new methodology is provided for parking planning. The model considers the parking choice at the end of the trip in modelling dynamic route choice behaviours of drivers, can give not only the number of vehicles going into or out of parking lots but also searching routes of vehicles for parking spaces, so it is suitable for evaluating the "ineffective traffic" and traffic congestion to surrounding networks caused by parking cruising. Therefore, changing generalized impedance involving time and expense of each route through changing parking charge of each park can change parking choices of vehicles, control cruising traffic and thus alleviate traffic congestion.

This paper intensively analyses flow propagation formula in the access passage of the parking lots. We believe this formula can be borrowed by flow propagation analysis for the congested road links. Traffic flows on the access passage of parking lots are like those on the congested links in road networks, so a similar analysis method can be applied there in the future work.

\section{ACKNOWLEDGEMENTS}

This work was supported by the science and technology project of Hunan Province, China under Grant [number 2015ZK3003], and the Research Fund for Young Scholars of Central South University under Grant [number 2014JSJJ015].

陈群, 博士, 通讯作者

E-mail: chenqun631@csu.edu.cn

地址：中国湖南省长沙市韶山南路22号中南大学交通运输

工程学院, 邮编410075

王艳, 博士生

E-mail: wangyanheroushi@163.com

地址: 中国湖南省长沙市中南大学公共管理学院,

邮编 410083

\section{驾驶员动态停车选择行为模型}

\section{摘要}

考虑时变的停车需求及停车泊位供给, 本文建立动态 停车选择行为模型, 模拟车辆驾驶员在各停车点的选择与 搜索过程。在本研究中, 每个停车场有且仅有一个进口通 道, 车辆通过入口通道进入与其相连的停车场, 车辆可在 停车场的入口通道内行驶或排队等待进入停车场。建立了 车辆在停车场 (库) 入口通道的车流流量传播方程, 以计 算每个时间间隔内从入口通道进入停车场（库）的车辆 数。提出了动态停车选择的瞬时动态用户最优条件, 并建 立了等价的变分不等式模型, 提出对角化算法对模型进行 求解。通过一个算例对模型与算法进行了验证，可得到各 个时刻各条路段及停车场入口通道的流入率、流出率、流 量、瞬时阻抗以及巡游交通流流量与流向。

\author{
关键词 \\ 停车; 动态; 用户最有; 变分不等式 $(V I)$
}




\section{REFERENCES}

[1] Mahamassani HS, Peeta S. Network performance under system optimal and user equilibrium dynamic assignment: implications for ATIS. Transportation Research Record. 1993 Aug;1408:83-93.

[2] Florian M, Mahut M, Tremblay N. Application of a simulation-based dynamic traffic assignment model. European Journal of Operational Research. 2008 Sep;189(3):1381-1392.

[3] Merchant DK, Nemhauser GL. A model and an algorithm for the dynamic traffic assignment. Transportation Science. 1978 Aug;12(3):183-199.

[4] Carey M. Optimal time-varying flows on congestion networks. Operations Research. 1987 Feb;35(1):58-69.

[5] Ran R, LeBlanc $\sqcup$, Boyce DE. Dynamic user-optimal route choice models based on stochastic route travel times. Second Int. Capri Seminar on Urban Traffic Networks; 1992.

[6] Lam WHK and Huang HJ. Dynamic user optimal traffic assignment model for many to one travel demand. Transportation Research B. 1995 Aug;29(4):243-259.

[7] Ran B, Boyce DE, LeBlanc L. A new class of instantaneous dynamic user-optimal traffic assignment models. Operations Research. 1993 Feb;41(1):192-202.

[8] Ran B, Boyce DE. Modeling dynamic transportation network: an intelligent transportation system oriented approach. Springer-Verlag Berlin and Heidelberg GmbH \& Co. K; 1996.

[9] Chen HK. Dynamic travel choice model: A variational inequality approach. Springer-Verlag Berlin and Heidelberg GmbH \& Co. K; 1999.
[10] Nie Y, Zhang HM. Solving the Dynamic User Optimal Assignment Problem Considering Queue Spillback. Networks \& Spatial Economics. 2010 March;10(1):49-71.

[11] Kaufman DE, Smith RL, Wunderlich KE. User-equilibrium properties of fixed points in dynamic traffic assignment. Transportation Research C. 1998 Feb;6(12):1-16.

[12] Lo HK, Szeto WY. A cell-based variational inequality formulation of the dynamic user optimal assignment problem. Transportation Research B. 2002 June;36(5):421-443.

[13] Ramadurai G, Ukkusuri S. Dynamic user equilibrium model for combined activity-travel choices using activity-travel supernetwork representation. Networks \& Spatial Economics. 2010 June;10(2):273-292.

[14] Tian L, Huang HJ, Gao ZY. A cumulative perceived value-based dynamic user equilibrium model considering the travelers' risk evaluation on arrival time. Networks \& Spatial Economics. 2012 Dec;12(4):589-608.

[15] Jiang Y, Wong SC, Ho HW, Zhang P, Liu R, Agachai S. A dynamic traffic assignment model for a continuum transportation system. Transportation Research B. 2011 Feb;45(2):343-363.

[16] Gao ZY, Ren HL. Urban dynamic traffic assignment model and algorithm [in Chinese]. Beijing: Renmin Jiaotong Press; 2005.

[17] Frank M, Wolfe P. An algorithm for quadratic programming. Naval Research Logistics Quarterly. 1956 June;3(2):95 -110.

[18] Sheffi Y. Urban Transportation Networks: Equilibrium Analysis with Mathematical Programming Methods. Prentice-Hall, Englewood Cliffs, N J; 1985. 